\title{
Should we screen for type 2 diabetes among asymptomatic individuals? Yes
}

\author{
David Simmons $^{1} \cdot$ Janice C. Zgibor $^{2}$
}

Received: 15 June 2017 / Accepted: 14 July 2017 /Published online: 23 August 2017

(C) Springer-Verlag GmbH Germany 2017

\begin{abstract}
RCTs of whether screening asymptomatic individuals for undiagnosed diabetes results in reduced mortality or has other benefits have been suggestive, but inconclusive. In this issue of Diabetologia, two additional controlled studies (DOIs: 10.1007/ s00125-017-4323-2 and 10.1007/s00125-017-4299-y) that investigated whether screening for type 2 diabetes in asymptomatic individuals is associated with a reduction in mortality are presented. Treating diabetes early, and identifying and treating impaired glucose tolerance, are of benefit, and economic modelling indicates such screening is cost-effective. Now that such screening is already underway in many countries, new data, along with the existing evidence, suggests opportunistic screening is the best way forward. More research is needed, however, on how best to screen and how to improve risk-factor control once dysglycaemia is detected.
\end{abstract}

Keywords Cardiovascular disease $\cdot$ Diabetes mellitus . Hospitalisation $\cdot$ Mortality primary care $\cdot$ Screening

\begin{abstract}
Abbreviations
ABPI Ankle-brachial pressure index

ADDITION Anglo-Danish-Dutch study of intensive treatment in people with screen-detected diabetes in primary care
\end{abstract}

David Simmons

da.simmons@westernsydney.edu.au

1 School of Medicine, Western Sydney University, Locked Bag 1797, Campbelltown, NSW 2751, Australia

2 Department of Epidemiology and Biostatistics, College of Public Health, University of South Florida, Tampa, FL, USA
CVD
Cardiovascular disease
GDM
IFG
IGT

\section{Introduction}

Screening for undiagnosed disease remains a 'hot topic'. Discussions continue over screening for various conditions; from cancer, with the risk of potentially harmful interventions in the falsely diagnosed, to gestational diabetes mellitus (GDM) and the need to balance diagnostic criteria with medicalising pregnancies, workload, and benefits to the mother and baby. In 2008, the growing importance of genomics, and the need to consider education and informed choice, led the World Health Organization to revise the original 1968 Wilson-Jungner's criteria to justify the introduction of systematic screening [1]. There has been a longstanding question mark over the effectiveness of systematic screening for type 2 diabetes, or whether to continue with case finding at times of hyperglycaemic symptoms or potentially diabetes-related events. Screening for diabetes responds to a recognised need to reduce the current high risk of complications. The objectives of screening are clear, as earlier management may prevent or delay the onset of diabetes and, therefore, its complications. However, there have been two main gaps in the evidence: whether screening is effective for improving outcomes and, if so, whether the benefits outweigh any harm. 'Harms' relate more to health service costs and potential impact of increased personal health insurance rather than psychological harm, which has been shown to be unlikely [2]. 


\section{Evidence from diabetes screening studies}

A systematic review in 2014, to update the 2008 US Preventive Services Task Force recommendations [3], concluded that, while treatment for impaired fasting glucose (IFG)/impaired glucose tolerance (IGT) delayed progression to (type 2) diabetes, screening for diabetes did not improve mortality rates. This conclusion was based upon two studies: (1) the Anglo-Danish-Dutch study of intensive treatment in people with screen-detected diabetes in primary care (ADDITION)-Cambridge trial ( $n=16,047$; high risk men and women, aged 40-69 years; 10 year duration) [4], with a mortality HR of 1.06 (95\% CI 0.90, 1.25); and (2) the Ely trial ( $n=4936$; all men and women aged 40-65 years; 18 year duration) [5]. The Ely trial included a 1990-1999 cohort (HR 0.79 [95\% CI $0.63,1.00]$ ) and a 2000-2008 cohort (HR 1.18 [95\% CI 0.93, 1.51]). The Ely trial also compared clinical characteristics between those diagnosed with diabetes who were randomised to its screening $(n=92)$ and non-screening $(n=60)$ limbs over 12 years [6]. Besides a significantly higher ankle-brachial pulse index (also known as ankle-brachial pressure index [ABPI] $)(p=0.035)$ and a non-significant increase in retinopathy in the unscreened group $(21 \%$ vs $35 \%$ [including one participant with proliferative retinopathy in the unscreened group]; $p=0.08$ ), clinical characteristics were similar.

Simmons et al have now presented two additional controlled studies in this issue of Diabetologia, which investigated whether screening for type 2 diabetes, in asymptomatic individuals, is associated with a reduction in mortality $[7,8]$. The studies were both part of the ADDITION-Europe study, but this time from the Danish limb (ADDITION-Denmark). One of these studies shows no reduction in mortality rate in participants in the screening programme vs the general Danish population [7], while the other [8] shows an association with participation in ADDITION and a significant reduction in adjusted HRs for all-cause mortality $(0.79$ [95\% CI $0.74,0.84])$, cardiovascular mortality $(0.80$ [95\% CI $0.72,0.88])$, cancer mortality $(0.83$ [95\% CI $0.77,0.89])$ and diabetes-related mortality (0.66 [95\% CI 0.54, 0.81]). The second study [8] also showed an association with a significant reduction in cardiovascular disease (CVD) events HR (0.84 [95\% CI 0.80, 0.89]). Another study, by Feldman et al, also shows reduced all-cause mortality, cardiovascular disease, renal disease and retinopathy [9] among those who were screened and had their diabetes diagnosed early. What are we to make of these discordant findings? Do the latter studies provide evidence that we should screen asymptomatic individuals for diabetes and, if so, who should this include and how should we screen?

\section{Limitations of ADDITION}

We would suggest that, unfortunately, none of the studies has been designed adequately to test whether we should screen asymptomatic individuals for diabetes. Only the Ely and ADDITION-Cambridge studies included unscreened, randomly-selected control participants, at least allowing a properly controlled comparison of outcomes. In contrast, the ADDITION-Denmark studies were non-randomised and compare mortality (and cardiovascular events) only among those with diabetes, excluding any benefits among those identified with IFG and/or IGT. Furthermore, comparing patients in practices who agreed to participate in a trial (i.e. participants of ADDITION-Denmark) with those in practices who were not invited or declined to take part (a proportion of the ADDITION-Denmark control participants) is particularly fraught with selection bias, as general practitioners who are interested in diabetes achieve better diabetes outcomes than those less interested [10]. The ADDITION-Cambridge and Ely studies both overcame this problem by including randomised practices, or randomised individuals within a single practice, respectively. However, both of these UK-based studies included only a small number of participants with diabetes; the ADDITION-Cambridge study was carried out across multiple practices but only a small proportion ( $n=466$ or $3 \%)$, actually had screen-detected diabetes [4]. Similarly, the Ely study included only 2699 screened participants over 12 years, with 199 (7\%) having newly-detected diabetes. The work by Feldman et al, suffered from the usually inherent potential selection biases.

The Ely study differed to the ADDITION studies in that all participants underwent an OGTT at baseline. ADDITION included a less comprehensive screening approach, using a risk calculator, then random capillary $\mathrm{HbA}_{1 \mathrm{c}}$ and glucose measurements using point of care meters, followed by a capillary fasting glucose test. Subsequently, an OGTT was carried out if screening was 'positive' by study criteria. The screened cohort in the Ely trial also had the OGTT repeated after approximately 5 years, while the ADDITION studies were based upon a single round of screening. Participant characteristics in each study therefore differed.

\section{The impact of post-screening management on diabetes outcomes}

Once diagnosed, a key factor determining mortality among those with diabetes is the intensity of the management received. Indeed, the ADDITION-Europe study was designed to compare the effect of intensive management with usual care for such patients, and after 5 years [11], overall it was found 
that intensive management was associated with only a small non-significant reduction in the incidence of cardiovascular events and death (HR 0.91 [95\% CI 0.69, 1.21]). However, there were substantial site differences, with a significant reduction in all-cause mortality across the two UK cohorts (Cambridgeshire and Leicester; HR 0.59 [0.35, 0.98]) but not in the Danish cohort. Danish healthcare differs to the English National Health Service and is ranked lower than the UK (as a whole) in a comparison of selected diabetes outcomes across the Organisation for Economic Co-operation and Development (OECD) [12]. This suggests that differences in care delivery may explain some of the differences in findings between ADDITION sites. Cambridgeshire (where both the ADDITION-Cambridge and Ely studies were undertaken) had patchy quality in local diabetes care and, hence, outcomes may have been even better with a more integrated approach to care [13]. However, overall, target achievement was only slightly greater in the practices that offered intensive intervention, so it may be that the intensive management plan used was insufficient to achieve a difference in CVD events vs control participants. We also know that intensive management takes time to achieve significant differences in mortality rates [14] and the ADDITION study may have been too short.

Conversely, overly intensive glycaemic management of type 2 diabetes is associated with increased mortality, albeit with reduced CVD events [15]. Since these screening trials were undertaken, diabetes treatment now includes agents that are associated with lower mortality (as determined by RCTs) $[16,17]$; hence, outcomes would be expected to be better if such new agents had been available at the time of the studies (and used appropriately).

Of course, diabetes management also involves aggressive management of cardiovascular risk factors, particularly dyslipidaemia and hypertension, which, as shown by these new ADDITION data, are more rapidly accessed in screen-detected diabetes [7]. Access to the appropriate pharmaceutical agents and use of appropriate therapeutic targets, with minimal clinical inertia, would, therefore, be required in any trial testing the effectiveness of screening for diabetes. This requirement was not a component of the Ely study.

\section{Designing future trials of diabetes screening}

As a result of the evidence to date, the design of any new RCT in diabetes screening must include a plan for treatment of any participants that are newly-diagnosed with IFG, IGT or diabetes in the study. In line with this, ethical considerations now prevent the use of blinded OGTTs or screening tests at
Lessons from diabetes screening studies

(1) Treating hyperglycaemia in type 2 diabetes from diagnosis reduces micro- and macrovascular outcomes and mortality over 10 years [14]

2 Managing IFG/IGT reduces progression to type 2 diabetes over 15 years [18]

3 Intensive management of screen-detected diabetes reduces incidence of cardiovascular events and death over 5 years in the UK [11]

4 In the Ely trial, testing for diabetes using an OGTT with repeated OGTT after 5 years was associated with non-significant reductions in all-cause mortality, significantly lower ABPI and non-significantly reduced rates of retinopathy in those in the screening group who were diagnosed with diabetes vs unscreened individuals $[5,6]$

In the 2015 US Preventive Services Task Force systematic review, all seven health economic modelling studies reported screening (vs no screening) at age $40-45$ is associated with incremental cost-effectiveness ratios of $<\$ 15,000$ /quality-adjusted life year (QALY), which is well under the traditional thresholds for cost-effectiveness [3]

the commencement of any trial. Because of this, the design of future RCTs in diabetes screening would require randomisation to either a screened or unscreened cohort (as per ADDITION-Cambridge). The numbers required for such a trial have now been calculated by Echouffo-Tcheugui and Prorok [19], based on ADDITION and other studies. They show that the number of people needed in each arm of a 10 year diabetes screening trial among adults aged 55-64 years, with $80 \%$ power to show a $15 \%$ reduction in mortality, would be 306,356 .

Furthermore, such a trial may no longer be viable in many countries that have now adopted national screening and prevention programmes (e.g. the UK), in which the yield of unscreened individuals with undiagnosed diabetes may be low. This may be the case particularly among women, many of whom, with [20] or without [21] diabetes risk factors, will now be screened for GDM using a blood test. In fact, the proportion of individuals with undiagnosed diabetes seems to be dropping already. For example, the latest UK modelling suggests that only $25 \%$ of those with diabetes are now undiagnosed [22], half of the $50 \%$ quoted historically. This 
is likely to be the result of opportunistic screening in primary care. These developments increasingly suggest that both policy makers and primary care practitioners believe that equipoise has now been reached for screening for undiagnosed diabetes, and the only questions that remain are who and how to screen. Whether to use a risk score followed by a 'blood test', or universal screening with a 'blood test' at, for example, 40-45 years of age, remains open. The use of a combination of random glucose levels $(5.5-11.0 \mathrm{mmol} / \mathrm{l})$ with an $\mathrm{HbA}_{1 \mathrm{c}} \geq 6.1 \%$ (43 $\left.\mathrm{mmol} / \mathrm{mol}\right)$, as used by ADDITION, is one approach to decide who should proceed to the next test of either fasting glucose alone, OGTT or $\mathrm{HbA}_{1 \mathrm{c}}$. The substantial problems with $\mathrm{HbA}_{1 \mathrm{c}}$ analysis, a test that is significantly influenced by the lifespan of the red blood cell, are that it generates multiple false positive and false negative tests [23], and is poor at detecting IGT/IFG without use of a low threshold (5.7\% [40 mmol/mol]) [24], suggesting that $\mathrm{HbA}_{1 \mathrm{c}}$ should not be used alone for screening for diabetes. A trial comparing different screening strategies, with different expected uptake (higher for random blood glucose and $\mathrm{HbA}_{1 \mathrm{c}}$ than fasting blood glucose or OGTT) and sensitivities, may be the next step in countries with national prevention/screening programmes already in place. Countries with national records, such as Sweden, would be ideal for such a study.

A trial of screening may be feasible and ethical in populations where the incidence of type 2 diabetes is high, the incidence of diabetes complications is also high (e.g. many non-European populations) and access to screening and quality diabetes care (once diagnosed) is limited. Such a trial could use a stepped-wedge cluster design [25], so that all participating communities would gain access to the intervention over time, including strategies to optimise care quality. There would need to be a commitment to establishing a sustainable screening and management programme for those with existing diabetes beyond the life of the trial and an associated prevention programme for those with IGT/IFG would need to be introduced. The power calculations of Echouffo-Tcheugui and Prorok would need to be reviewed, with a potential for the required numbers to be reduced as they are based upon European populations with lower prevalence of diabetes and complication rates, although this may be mitigated by the falling CVD rates with modern therapy. Even so, this would require substantial investment in both materials and staff, and must be able to fit into the existing healthcare system.

\section{Conclusions}

In conclusion, trials of screening for undiagnosed diabetes among asymptomatic individuals may no longer be feasible or ethical in many countries. The most efficient recommendation may be opportunistic screening [26], where patients already seeking care (including screening) for another condition are subsequently tested for diabetes or IGT/IFG. If screened positive, they are more likely to receive treatment, thus leading to improved outcomes. There is probably sufficient evidence to conclude that this systematic approach to screening should occur in primary care (i.e. not through a centralised approach, as occurs for some cancers) and that focus should now shift to trials of how to screen, methods for implementing treatment earlier, and better risk factor control in those at highest risk.

Funding Work by DS is supported by South Western Sydney Local Health District and Western Sydney University.

Duality of interest The authors declare that there is no duality of interest associated with this manuscript.

Contribution statement Both authors were responsible for drafting the article and revising it critically for important intellectual content. Both authors approved the version to be published.

\section{References}

1. Andermann A (2008) Revisiting Wilson and Jungner in the genomic age: a review of screening criteria over the past 40 years. Bull World Health Organ 86:317-319

2. Eborall HC, Griffin SJ, Prevost AT et al (2007) Psychological impact of screening for type 2 diabetes: controlled trial and comparative study embedded in the ADDITION (Cambridge) randomised controlled trial. BMJ 335:486

3. Dall TM, Narayan KMV, Gillespie KB et al (2014) Detecting type 2 diabetes and prediabetes among asymptomatic adults in the United States: modeling American Diabetes Association versus US Preventive Services Task Force diabetes screening guidelines. Popul Health Metrics 12:12

4. Simmons RK, Echouffo-Tcheugui JB, Sharp SJ et al (2012) Screening for type 2 diabetes and population mortality over 10 years (ADDITION-Cambridge): a cluster-randomised controlled trial. Lancet 380:1741-1748

5. Simmons RK, Rahman M, Jakes RW et al (2011) Effect of population screening for type 2 diabetes on mortality: long-term followup of the Ely cohort. Diabetologia 54:312-319

6. Rahman M, Simmons RK, Hennings SH et al (2012) How much does screening bring forward the diagnosis of type 2 diabetes and reduce complications? Twelve year follow-up of the Ely cohort. Diabetologia 55:1651-1659

7. Simmons RK, Griffin SJ, Witte DR et al (2017) Effect of population screening for type 2 diabetes and cardiovasular risk factors on mortality rate and cardiovascular events: a controlled trial among 1,912, 392 Danish adults. Diabetologia doi:10.1007/s00125-017-4323-2

8. Simmons RK, Griffin SJ, Lauritzen T, Sandbæk A (2017) Effect of screening for type 2 diabetes on risk of cardiovascular disease and mortality: a controlled trial among 139,075 individuals diagnosed with diabetes in Denmark between 2001 and 2009. Diabetologia doi:10.1007/s00125-017-4299-y

9. Feldman AL, Griffin SJ, Fhärm E et al (2017) Screening for type 2 diabetes: do screen-detected cases fare better? Diabetologia doi:10.1007/s00125-017-4402-4 
10. Pringle M, Stewart-Evans C, Coupland C, Williams I, Allison S, Sterland J (1993) Influences on control in diabetes mellitus: patient, doctor, practice, or delivery of care? BMJ 306:630-634

11. Griffin SJ, Borch-Johnsen K, Davies MJ et al (2011) Effect of early intensive multifactorial therapy on 5-year cardiovascular outcomes in individuals with type 2 diabetes detected by screening (ADDITION-Europe): a cluster-randomised trial. Lancet 378: 156-167

12. OECD (2015) Health at a Glance 2015: OECD Indicators, OECD Publishing, Paris, pp134-135. Available from http://dx. doi.org/10.1787/health_glance-2015-en. Accessed 3 July 2017

13. Simmons D, Yu D, Bunn C, Cohn S, Wenzel H, Prevost T (2015) Hospitalization among patients with diabetes associated with a diabetes impact initiative: a mixed methods case study. Future Hospital J 2:92-98

14. Holman RR, Paul SK, Bethel MA et al (2008) 10-year follow-up of intensive glucose control in type 2 diabetes. N Engl J Med 359: $1577-1589$

15. The Action to Control Cardiovascular Risk in Diabetes Study Group (2008) Effects of intensive glucose lowering in type 2 diabetes. N Engl J Med 358:2545-2559

16. Zinman B, Wanner C, Lachin JM et al (2015) Empagliflozin, cardiovascular outcomes, and mortality in type 2 diabetes. $\mathrm{N}$ Engl $\mathrm{J}$ Med 373:2117-2128

17. Marso SP, Daniels GH, Brown-Frandsen K et al (2016) Liraglutide and cardiovascular outcomes in type 2 diabetes. N Engl J Med 375: 311-322

18. Diabetes Prevention Research Group (2016) Long-term effects of lifestyle intervention or metformin on diabetes development and microvascular complications over 15-year follow-up: the Diabetes Prevention Program Outcomes study. Lancet Diabetes Endocrinol 3:866-875
19. Echouffo-Tcheugui JB, Prorok PC (2014) Considerations in the design of randomized trials to screen for type 2 diabetes. Clin Trials 11:284-291

20. National Institute for Health and Care Excellence (NICE) guidelines (2015). Diabetes in pregnancy: management of diabetes and its complications from preconception to the postnatal period (NG3). Available from www.nice.org.uk/guidance/ng3/resources/diabetesin-pregnancy-management-of-diabetes-and-its-complicationsfrom-preconception-to-the-postnatal-period-51038446021. Accessed 1 April 2015

21. Nankervis A, McIntyre HD, Moses R, et al (2014) ADIPS consensus guidelines for the testing and diagnosis of hyperglycaemia in pregnancy in Australia and New Zealand. Available from http:// adips.org/downloads/2014ADIPSGDMGuidelinesV18.11.2014 000.pdf. Accessed 2 Jan 2017

22. Public Health England (2015) Diabetes prevalence estimates for local populations. Available from www.gov.uk/government/ publications/diabetes-prevalence-estimates-for-local-populations. Accessed 15 June 2017

23. Church D, Simmons D (2014) More evidence of the problems of using $\mathrm{HbAlc}$ for diagnosing diabetes? The known knowns, the known unknowns and the unknown unknowns. J Intern Med 276: $171-173$

24. Waugh NR, Shyangdan D, Taylor-Phillips S, Suri G, Hall B (2013) Screening for type 2 diabetes: a short report for the National Screening Committee. Health Technol Assess 17:1-90

25. Brown CA, Lilford RJ (2006) The stepped wedge trial design: a systematic review. BMC Med Res Methodol 6:54

26. Engelgau MM, Narayan KM, Herman WH (2000) Screening for type 2 diabetes. Diabetes Care 23:1563-1580 